\title{
Effect of clinical symptoms on the indication for selective neck dissection for NO carcinomas of the parotid gland
}

\author{
TAKASHI MARUO, YASUSHI FUJIMOTO, KENJI YOSHIDA, MARIKO HIRAMATSU, \\ ATSUSHI SUZUKI, NAOKI NISHIO, MARIKO SHIMONO and TSUTOMU NAKASHIMA
}

Department of Otorhinolaryngology, Nagoya University Graduate School of Medicine, Nagoya, Aichi 466-8550, Japan

Received September 7, 2013; Accepted April 10, 2014

DOI: $10.3892 / \mathrm{ol} .2014 .2137$

\begin{abstract}
Lymph node metastasis is a major prognostic factor in parotid carcinoma, however, the pre-operative diagnosis of occult nodal metastasis is difficult in clinical NO (cN0) parotid cancer patients. In addition, the indication of neck dissection in T1-3 cN0 patients is controversial. The current study investigated 17 patients with clinical T1-3 cN0 parotid cancer, and analyzed the correlation between patient symptoms/findings and pathological $\mathrm{N}$ status/tumor histological grade. In the statistical analysis, pain was found to significantly correlate with neck metastasis. Furthermore, cN0-staged patients without pain exhibited no neck metastasis. However, no significant correlation was identified between patient symptoms or findings and histological grade. These results indicate the possibility that selective neck dissection can be omitted for T1-3 cN0-staged patients without pain.
\end{abstract}

\section{Introduction}

Carcinoma of the parotid gland represents $\sim 2 \%$ of all head and neck cancers (1). Cancer of the parotid gland is classified into several histological types, and the grade of parotid cancer varies with the histological type. As a result, certain tumors are slow growing, while others are more aggressive. Treatment is typically surgery, which may be followed by radiation therapy, while chemotherapy can be effective in treating later stage cancers.

Cervical nodal metastases are a major adverse prognostic factor $(2,3)$. High tumor grade, extraparotid extension, a tumor size of $\geq 4 \mathrm{~cm}$ and facial nerve involvement are associated with nodal disease (4). Even if the option of selective neck dissection is determined on the basis of histological grade or primary tumor stage ( $\mathrm{T}$ stage), an accurate pre-operative assessment of the histological grade may be difficult in

Correspondence to: Dr Yasushi Fujimoto, Department of Otorhinolaryngology, Nagoya University Graduate School of Medicine, 65 Tsurumai-cho, Nagoya, Aichi 466-8550, Japan

E-mail: tmaruo@med.nagoya-u.ac.jp

Key words: parotid gland cancer, neck dissection, clinical symptoms patients with parotid carcinoma $(5,6)$. In previous studies, it has been reported that more than half of T4-staged parotid carcinoma patients exhibit neck node metastasis (6). As a result, we recommend surgery with elective neck dissection (END) for T4-staged parotid carcinoma. However, the treatment of T1-T3-staged patients remains controversial.

The National Comprehensive Cancer Network guidelines (7) show that the characteristics of a benign tumor include a mobile superficial lobe, slow growth and no pain, as well as a lack of or intact neck nodes. This has shown that in addition to neck nodes and facial nerve paralysis, clinical symptoms are also significant. The present study was conducted to determine the correlation between clinical symptoms and nodal metastasis and clinical outcome in patients with T1-3 parotid cancers. The specific point of interest was the investigation of the pretreatment clinical symptoms of regional lymph node stage ( $\mathrm{N}$ stage), and in particular clinical N0 (cN0)-staged patients at a high risk for occult metastasis who may potentially benefit from selective neck treatment.

\section{Patients and methods}

Population data. Between 2003 and 2011, 35 previously untreated patients with carcinoma of the parotid gland received definitive treatment at the Nagoya University Hospital (Nagoya, Japan). In the present study, 17 T1-3-staged patients (Fig. 1, Table I) of the 35 patients were analyzed according to the inclusion critearia of $\mathrm{T}$ and $\mathrm{N}$ stage, including 11 males and six females who ranged in age between 27 and 80 years. The median follow-up duration was 47 months. T and $\mathrm{N}$ staging, histological type and four clinical findings (tumor mobility, neck pain, facial palsy and skin invasion) were analyzed. The clinical findings were compared with the presence of lymph node metastasis and histological type grade. Patients provided written informed consent.

TN staging, histological grades and diagnosis. Seven of the 17 patients (41.2\%) were classified as T1, six (35.3\%) as T2 and four $(23.5 \%)$ as T3 (Table I). In addition, 15 patients were regarded as cN0 and two as N2b (Fig. 1). Selective neck dissection was performed for all surgical parotid cancer patients, including stage NO patients. The histological grade classified patients into three groups: High, intermediate and low grade. 
Table I. Analysis of patients with parotid cancer.

\begin{tabular}{clccc}
\hline Patient no. & Gender & Age, years & T stage & N stage \\
\hline 1 & Male & 72 & 2 & 0 \\
2 & Male & 65 & 3 & 0 \\
3 & Male & 44 & 1 & 0 \\
4 & Male & 62 & 1 & 0 \\
5 & Female & 60 & 2 & 0 \\
6 & Male & 53 & 2 & 0 \\
7 & Male & 59 & 3 & $2 \mathrm{~b}$ \\
8 & Male & 80 & 2 & 0 \\
9 & Male & 58 & 3 & $2 \mathrm{~b}$ \\
10 & Female & 42 & 2 & 0 \\
11 & Female & 43 & 2 & 0 \\
12 & Male & 65 & 1 & 0 \\
13 & Male & 27 & 1 & 0 \\
14 & Female & 75 & 1 & 0 \\
15 & Male & 64 & 1 & 0 \\
16 & Female & 65 & 1 & 0 \\
17 & Female & 66 & 3 & 0 \\
\hline
\end{tabular}

Table II. Histological types and grade classified into three groups.

\begin{tabular}{cll}
$\begin{array}{l}\text { Patient } \\
\text { no. }\end{array}$ & \multicolumn{1}{c}{ Histological type } & \\
\hline 1 & Adenoid cystic carcinoma & \multicolumn{1}{c}{ Grade } \\
2 & Adenoid cystic carcinoma & Intermediate \\
3 & Adenoid cystic carcinoma & Intermediate \\
4 & Mucoepidermoid carcinoma & Intermediate \\
5 & Carcinoma ex pleomorphic adenoma & Low \\
6 & Mucoepidermoid carcinoma & Low \\
7 & Salivary duct carcinoma & High \\
8 & Epithelial myoepithelial carcinoma & Intermediate \\
9 & Salivary duct carcinoma & High \\
10 & Mucoepidermoid carcinoma & Intermediate \\
11 & Adenoid cystic carcinoma & Intermediate \\
12 & Adenocarcinoma NOS & Low \\
13 & Epithelial myoepithelial carcinoma & Intermediate \\
14 & Mucoepidermoid carcinoma & Low \\
15 & Adenocarcinoma NOS & Low \\
16 & Mucoepidermoid carcinoma & Intermediate \\
17 & Adenoid cystic carcinoma & Low \\
& &
\end{tabular}

NOS, not otherwise specified.

Of the 17 patients, two patients were classified as high, eight (47.1\%) as intermediate and seven $(41.2 \%)$ as low grade. Each histological type for all the patients and the grade-dependent classification are shown in Table II.

Evaluation of clinical symptoms and findings. All the symptoms of the T1-3 N0 patients $(n=15)$ were analyzed, and tumor
Table III. Patient findings and symptoms.

\begin{tabular}{ll}
\hline Clinical symptoms & $\mathrm{n}$ \\
\hline Pain $(\mathrm{n}=15)$ & 5 \\
Poor mobility $(\mathrm{n}=11)$ & 7 \\
Skin invasion $(\mathrm{n}=15)$ & 0
\end{tabular}

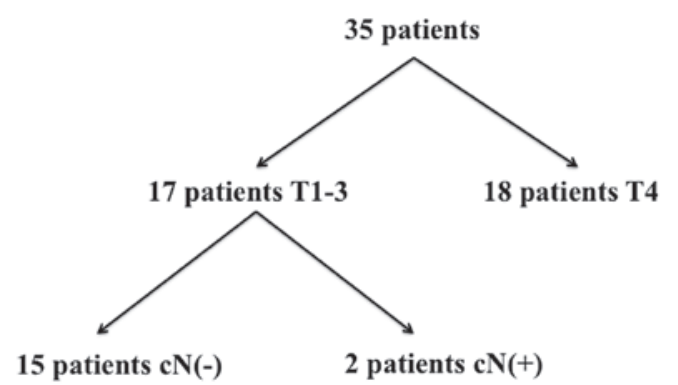

Figure 1. Parotid cancer patient characteristics and pre-operative clinical staging. cN, clinical $\mathrm{N}$ stage.

mobility was analyzed in 11 patients. As the clinical reports of four patients did not contain adequate data, they were excluded from the analysis. In total, five of the 11 patients exhibited poor tumor mobility, and five out of 15 patients exhibited pain. None of the patients exhibited facial palsy or skin invasion (Table III). The correlation between these symptoms, and LN metastasis and histological grade were evaluated.

Treatment. All patients were initially treated by parotid resection. Neck dissection was performed on all patients, including NO patients, in which levels I, II and III were resected and examined histologically. Facial nerves without tumor invasion were preserved when possible. Post-surgical irradiation was performed in five patients $(29.4 \%)$ with a high-grade tumor or positive surgical margin.

Statistical analysis. The statistical analysis was performed using Pearson's $\chi^{2}$ test, and the survival expectation was calculated by the Kaplan-Meier test. $\mathrm{P}<0.05$ was considered to indicate a statistically significant difference. All statistical analyses were performed using JMP 8.0 software (SAS Institute, Inc., Cary, NC, USA).

\section{Results}

Pathological staging. According to the histological examination of the neck node at the pretreatment examination, 13 patients were determined as pathological N0 (pN0), 15 patients as cN0 and two patients as pN1 (Table IV).

Comparison between clinical findings and neck metastases. Three patients presented with pain, but without neck metastases, while two patients presented with neck metastasis. In addition, 10 patients were without pain and neck metastasis. No patients were identified without pain, but with neck metastasis. A significant correlation was identified between pain and neck metastasis $(\mathrm{P}<0.05$; Table $\mathrm{V})$. 
Table IV. N staging following pathological examination of resected lymph node ( $\mathrm{pN})$.

\begin{tabular}{lcccrc}
\hline & \multicolumn{4}{c}{$\mathrm{pN}$ status, $\mathrm{n}$} \\
\cline { 2 - 6 } $\mathrm{cN}$ status & $\mathrm{pN0}$ & $\mathrm{pN} 1$ & $\mathrm{pN} 2$ & $\mathrm{pN} 3$ & Total \\
\hline $\mathrm{cN0}$ & 13 & 2 & 0 & 0 & 15 \\
\hline
\end{tabular}

$\mathrm{pN}$, pathological $\mathrm{N}$ stage; $\mathrm{cN}$, clinical $\mathrm{N}$ stage.

Table V. Symptoms compared with pN status.

A, Pre-operative pain symptoms

\begin{tabular}{lccr}
\hline Variable & Neck metastasis (+), & Neck metastasis (-), & Total, $\mathrm{n}$ \\
\hline Pain & 2 & 3 & 5 \\
No pain & 0 & 10 & 10 \\
Total & 2 & 13 & 15 \\
\hline
\end{tabular}

B, Pre-operative tumor mobility

\begin{tabular}{|c|c|c|c|c|}
\hline Variable & Neck metastasis $(+), \mathrm{n}$ & Neck metastasis $(-), n$ & Total, $\mathrm{n}$ & P-value ${ }^{a}$ \\
\hline Poor mobility & 2 & 3 & 5 & 0.08 \\
\hline Good mobility & 0 & 6 & 6 & \\
\hline Total & 2 & 9 & 11 & \\
\hline
\end{tabular}

${ }^{\text {aPearson's }} \chi^{2}$ test. $\mathrm{pN}$, pathological $\mathrm{N}$ stage.

Table VI. Pain and mobility symptoms compared with histological grade.

\begin{tabular}{lcccc}
\hline Grade & Pain, $\mathrm{n}$ & No pain, $\mathrm{n}$ & Poor mobility, $\mathrm{n}$ & Good mobility, $\mathrm{n}$ \\
\hline Low & 2 & 5 & 2 & 4 \\
Intermediate & 3 & 5 & 3 & 2 \\
High & 0 & 0 & 0 & 0 \\
\hline
\end{tabular}

Six patients with good tumor mobility were without neck metastasis, while no patients were identified with neck metastasis. Three of the 11 patients exhibited poor mobility of the tumor without neck metastasis, and two patients with poor mobility and neck metastasis. No significant correlation was identified between the mobility of the tumor and neck metastasis $(\mathrm{P}=0.08$; Table $\mathrm{V})$.

Comparison between clinical findings and histological grade. Two patients with pain had a low histological grade tumor, while three patients had an intermediate grade tumor. In addition, five patients without pain had a low histological grade tumor and five patients had an intermediate grade tumor. No significant correlation was identified between pain symptoms and the histological tumor grade $(\mathrm{P}=0.14$; Table $\mathrm{VI})$.
Furthermore, four patients with good mobility of the tumor had a low histological grade tumor and two patients had an intermediate grade tumor. Two patients presented with poor tumor mobility and a low histological grade tumor, and three with an intermediate grade tumor. No significant correlation was identified between the mobility of the tumor and the histological tumor grade $(\mathrm{P}=0.37$; Table $\mathrm{VI})$.

\section{Discussion}

Previous studies have suggested several prognostic factors for parotid carcinoma. In particular, histological grade and $\mathrm{T}$ and $\mathrm{N}$ staging have been analyzed in a number of studies (8-12). In the present study, $\mathrm{N}$ staging was evaluated as a prognostic factor, and it was considered whether END should be added to 
the parotid resection. It is commonly accepted that END must be performed in patients with a histologically high-grade malignancy, T3 or higher stage, facial palsy or extraparotid invasion, however, the indication of neck dissection is controversial (5).

The incidence of cervical nodal disease in parotid carcinoma is $14-16 \%$ (13). High tumor grade, extraparotid extension, a tumor size of $\geq 4 \mathrm{~cm}$, pain and facial nerve involvement are associated with nodal disease $(13,14)$.

Stodulski et al (15) analyzed the clinical signs and symptoms (facial palsy, skin invasion, neck lymphadenopathy, pain, tumor fixation and rapid tumor growth) as prognostic factors, and as a result, concluded that facial nerve palsy and skin infiltration are significant independent prognostic factors. In the current study, the patient findings and symptoms were considered to be of possible prognostic value for $\mathrm{N}$ status. Therefore, pain, tumor mobility, facial palsy and skin invasion of the tumor were analyzed.

In these analyses, only pain exhibited a significant correlation with $\mathrm{N}$ status. In previous studies, pain symptoms have shown no significant prognostic value (15). The current study is the first to report that pain symptoms exhibit a significant correlation with patients with or without neck metastasis. In particular, no patients without pain were identified with neck metastasis. This result indicated that $\mathrm{T} 1-3, \mathrm{cN} 0$-staged parotid carcinoma patients without pain may be treated by parotid resection only, without END. Other studies have also reported that pain symptoms can be divided into two types; earache and headache $(16,17)$. The current study did not investigate pain in this manner and therefore, these types of pain must be analyzed in the future.

A number of studies have also reported that histological grade is a significant prognostic factor $(1,10,11,18)$. However, histological types and grades are difficult to evaluate prior to surgery. The histological grades are often classified into three types (high, intermediate and low grade), and have show significant differences in prognosis in previous studies $(1,19,20)$. However, in the present study, no significant difference was identified between pain symptoms and tumor grade. The lack of high-grade tumors in T1-3, N0 patients may have led to this result and thus, follow-up and analysis are required.

The results of the current study indicate that T1-3, cNO-staged patients without pain exhibit no neck metastasis. It may be possible that selective neck dissection can be omitted for T1-3, cN0-staged patients without pain. By contrast, T1-3, cN0-staged patients with pain may exhibit occult neck metastasis and a poor prognosis. In conclusion, pain may be the only prognostic symptom that is useful in the pretreatment diagnosis of parotid carcinoma.

\section{Acknowledgements}

This study was supported by the Grants-in-Aid from the Research Committee of the Development and Standardization of Surgical Techniques in the Head and Neck Region, Japan (grant no. 23-A-26).

\section{References}

1. Spiro RH: Salivary neoplasms: overview of a 35-year experience with 2,807 patients. Head Neck Surg 8: 177-184, 1986.

2. Carrillo JF, Vázquez R, Ramírez-Ortega MC, et al: Multivariate prediction of the probability of recurrence in patients with carcinoma of the parotid gland. Cancer 109: 2043-2051, 2007.

3. Bhattacharyya N and Fried MP: Nodal metastasis in major salivary gland cancer: predictive factors and effects on survival. Arch Otolaryngol Head Neck Surg 128: 904-908, 2002.

4. Régis De Brito Santos I, Kowalski LP, Cavalcante De Araujo V, Flávia Logullo A and Magrin J: Multivariate analysis of risk factors for neck metastases in surgically treated parotid carcinomas. Arch Otolaryngol Head Neck Surg 127: 56-60, 2001.

5. Medina JE: Neck dissection in the treatment of cancer of major salivary glands. Otolaryngol Clin North Am 31: 815-822, 1998.

6. Kawata R, Koutetsu L, Yoshimura K, Nishikawa S and Takenaka H: Indication for elective neck dissection for N0 carcinoma of the parotid gland: a single institution's 20-year experience. Acta Otolaryngol 130: 286-292, 2010.

7. National Comprehensive Cancer Network: Clinical Practice Guidelines in Oncology ${ }^{\mathrm{TM}}$. Head and Neck Cancer Version 1.2012. http://www.nccn.org/professionals/physician_ gls/f_guidelines.asp. Accessed April 26, 2012.

8. Hocwald E, Korkmaz H, Yoo GH, et al: Prognostic factors in major salivary gland cancer. Laryngoscope 111: 1434-1439, 2001.

9. Lima RA, Tavares MR, Dias FL, et al: Clinical prognostic factors in malignant parotid gland tumors. Otolaryngol Head Neck Surg 133: 702-708, 2005.

10. Spiro RH, Huvos AG, Berk R and Strong EW: Mucoepidermoid carcinoma of salivary gland origin. A clinicopathologic study of 367 cases. Am J Surg 136: 461-468, 1978.

11. Hicks MJ, el-Naggar AK, Flaitz CM, Luna MA and Batsakis JG: Histocytologic grading of mucoepidermoid carcinoma of major salivary glands in prognosis and survival: a clinicopathologic and flow cytometric investigation. Head Neck 17: 89-95, 1995.

12. Frankenthaler RA, Luna MA, Lee SS, et al: Prognostic variables in parotid gland cancer. Arch Otolaryngol Head Neck Surg 117: 1251-1256, 1991.

13. Armstrong JG, Harrison LB, Thaler HT, et al: The indications for elective treatment of the neck in cancer of the major salivary glands. Cancer 69: 615-619, 1992.

14. Kelley DJ and Spiro RH: Management of the neck in parotid carcinoma. Am J Surg 172: 695-697, 1996.

15. Stodulski D, Mikaszewski B and Stankiewicz C: Signs and symptoms of parotid gland carcinoma and their prognostic value. Int J Oral Maxillofac Surg 41: 801-806, 2012.

16. Pohar S, Gay H, Rosenbaum P, et al: Malignant parotid tumors: presentation, clinical/pathologic prognostic factors, and treatment outcomes. Int J Radiat Oncol Biol Phys 61: 112-118, 2005.

17. Rafla S: Malignant parotid tumors: natural history and treatment. Cancer 40: 136-144, 1977.

18. Fordice J, Kershaw C, El-Naggar A and Goepfert H: Adenoid cystic carcinoma of the head and neck: predictors of morbidity and mortality. Arch Otolaryngol Head Neck Surg 125: 149-152, 1999.

19. Pedersen D, Overgaard J, Søgaard H, Elbrønd O and Overgaard M: Malignant parotid tumors in 110 consecutive patients: treatment results and prognosis. Laryngoscope 102: 1064-1069, 1992.

20. Therkildsen MH, Christensen M, Andersen LJ, Schiødt T and Hansen HS: Salivary gland carcinomas - prognostic factors. Acta Oncol 37: 701-713, 1998. 